\title{
Évolution in vitro des miracidiums de Scbistosoma mansoni
}

\author{
par J. BENEX et G. JACOBELLI \\ Institut Pasteur, Unité de Parasitologie expérimentale, 25, rue du Docteur-Roux, \\ F 75015 Paris.
}

RESUME. Des miracidiums de $S$. mansoni éclos et recueillis stérilement sont maintenus en culture dans un milieu nutritif dérivant de celui de Schneider et évoluent in vitro jusqu'au stade de sporocystes primaires âgés, parfaitement constitués. Cette évolution s'accompagne d'un remaniement membranaire comparable à celui observé in vivo.

\section{In vitro $\mathrm{S}$. mansoni miracidia transformation.}

SUMMARY. Miracidia de S. mansoni hatched and collected aseptically are able to survive in vitro in suitable media. Development of the miracidia is observed in the same manner that occurs in vivo. They shed their ciliated epiderma cell and miracidium-sporocyst transformation occurs. $30 \%$ of the miracidia explanted are transformed in primary sporocystes into $48 \mathrm{~h}$. and are still alive a week later.

Parmi les tentatives de culture in vitro des helminthes, un certain nombre concerne les Schistosomes. Mais la plupart ont porté sur les stades évoluant chez les mammifères.

Dans le cadre de nos études relatives à la spécificité hôte-parasite, nous nous sommes intéressés, pour notre part, depuis un certain temps déjà, aux stades évoluant chez l'hôte invertébré. Pour ce faire, nous disposons d'un matériel expérimental que nous connaissons bien : le couple Schistosoma mansoni-Biomphalaria glabrata entretenu au laboratoire depuis de nombreuses années.

Accepté le 23 juin 1980. 
La première expérimentation relative au développement in vitro des stades «Mollusques » est due à Barlow, en 1925, qui obtient le début de transformation en redies des miracidiums de Fasciolopsis buski (2).

Il faut attendre les années 1955/56 pour retrouver des essais de survie de miracidium in vitro à partir de Fasciola hepatica pour Campbell et Todd (5) et de Dicrostelium microsporum pour Ingersoll (7) avec des succès très limités.

Enfin Targett et Robinson (10), en 1964, travaillant avec les miracidiums de Schistosoma mansoni, observent des survies de 24/36 heures, avec une seule fois une ébauche de transformation de miracidium en sporocyste dans un milieu physiologique additionné d'extrait tissulaire de mollusques.

Les seuls résultats réellement positifs sont ceux rapportés d'une part par Voge et Seidel (11) en 1972 et Basch et Diconza (5) en 1974. Ces derniers auteurs étudièrent les modifications ultrastructurales de la membrane du miracidium lors de sa transformation en sporocyste primaire en milieu synthétique.

Pour notre part, nous désirions explorer les possibilités de défenses immunologiques des mollusques, au niveau cellulaire, à une infestation par des miracidiums de Schistosomes. Cette étude devant être conduite in vitro, nous avons voulu déterminer les possibilités de survie et d'évolution des miracidiums in vitro.

\section{Matériel}

Dans notre expérimentation nous avons donc utilisé une souche portoricaine de Schistosoma mansoni entretenue sur Biomphalaria glabrata et souris Swiss OF1; les foies de souris sont prélevés stérilement puis broyés et lavés en eau physiologique et eau distillée stériles additionnées de Pénicilline 100 U.I./ml, Colimycine 100 U.I./ml, Gentamicine $20 \mu \mathrm{g} / \mathrm{ml}$. L'éclosion des miracidiums se fait en flacon de Mac Mullen stérile dont seule la tubulure latérale est éclairée.

Dès leur éclosion, les miracidiums sont prélevés stérilement à la pipette Pasteur sous hotte à flux laminaire et répartis dans les flacons de culture contenant le milieu de survie.

Deux milieux ont été essayés :

\section{1. - Milieu de Schneider modifié :}

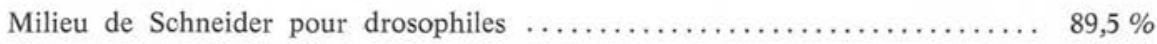

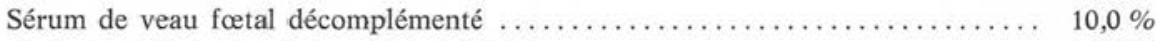

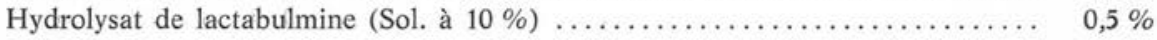

Pénicilline

Colimycine $\{100$ U.I./ml

Gentamicine $\quad 20 \mu \mathrm{g} / \mathrm{ml}$

Econazole $\quad 10 \mu \mathrm{g} / \mathrm{ml}$ 


\section{2. - Milieu de Hansen :}

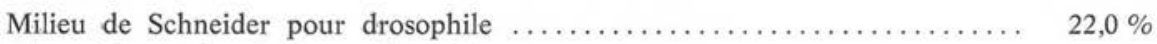

Sérum de veau fœetal décomplémenté ............................. 13,0\%

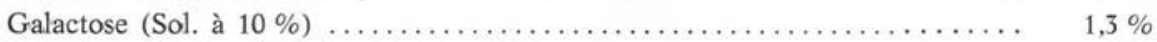

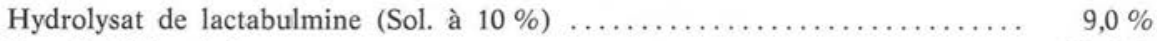

Eau distillée stérile q.s.p. .................................. $100,0 \%$

$\left.\begin{array}{l}\text { Pénicilline } \\ \text { Colimycine }\end{array}\right\} 100$ U.I./ml

Gentamicine $\quad 20 \mu \mathrm{g} / \mathrm{ml}$

Econazole $\quad 10 \mu \mathrm{g} / \mathrm{ml}$

Dans des flacons (Falcon) de $25 \mathrm{ml}$ contenant $5 \mathrm{ml}$ de milieu, on introduit 20 miracidiums fraîchement éclos maintenus sous atmosphère $8 \% \mathrm{CO}_{2}$; l'évolution de la survie est observée tous les jours sous microscope inversé. Le milieu n'est pas renouvelé.

\section{Résultats}

\section{Evolution des miracidiums.}

Introduits dans le flacon de culture, les miracidiums s'immobilisent rapidement. Une heure après, ils sont tous immobiles, leurs cils continuant à battre. Peu à peu ils s'en dépouillent. Les plaques ciliaires se détachent une à une et flottent dans le milieu liquide.

Durant toute cette période les miracidiums, bien qu'immobiles, font de nombreuses contractions. Au $4^{\mathrm{e}}$ jour, ils ont beaucoup allongé (environ le double de la longueur initiale). La membrane est parfaitement lisse.

Vers le $5^{\mathrm{e}}-6^{\mathrm{e}}$ jour, les contractions diminuent en intensité, les miracidiums ont évolué en sac allongé à paroi lisse empli de cellules germinatives et de réserves. Leur aspect est tout à fait similaire à celui décrit in vivo par Maldonado et Schutte (8). Dimensions : 400/600 $\mu \mathrm{m}$ de long sur $40 \mu \mathrm{m}$ de large. L'évolution se poursuit ainsi jusqu'au $19^{e}$ jour. A partir de ce moment celle-ci paraît stoppée, le contenu prend un aspect granuleux avec des cellules moins différenciées. Les sporocystes demeurent ainsi quelques jours mais n'évoluent plus. Ils ont alors une taille de 130/190 $\mu \mathrm{m}$.

\section{Influence du milieu.}

Les deux milieux de survie utilisés ont la même base définie par Schneider mais adaptée aux besoins physiologiques des Schistosomes. Cependant le milieu de Hansen, un peu plus riche en facteurs de croissance, permet un meilleur développement en taux de rendement aussi bien qu'en durée de survie. La différence en concentration ionique des deux milieux conditionne également le temps de latence pour l'immobilisation complète des miracidiums. Dans le milieu de Schneider, l'immobilisation a lieu plus rapidement et plus complètement dès la première heure que dans le milieu 
de Hansen où la motilité est conservée plus longtemps $(4 \mathrm{~h})$. En moyenne, on peut établir que sur 100 miracidiums mis en culture, 30 perdent leurs plaques ciliées; parmi ceux-ci, 15 évoluent jusqu'au stade sporocystes primaires.

Parmi ceux qui n'évoluent pas, certains ne perdent pas du tout leurs plaques ciliées ou très partiellement. D'autres présentent des réactions membranaires avec formation de bulles et d'excroissances en surface. Dans les deux cas, l'aspect extérieur
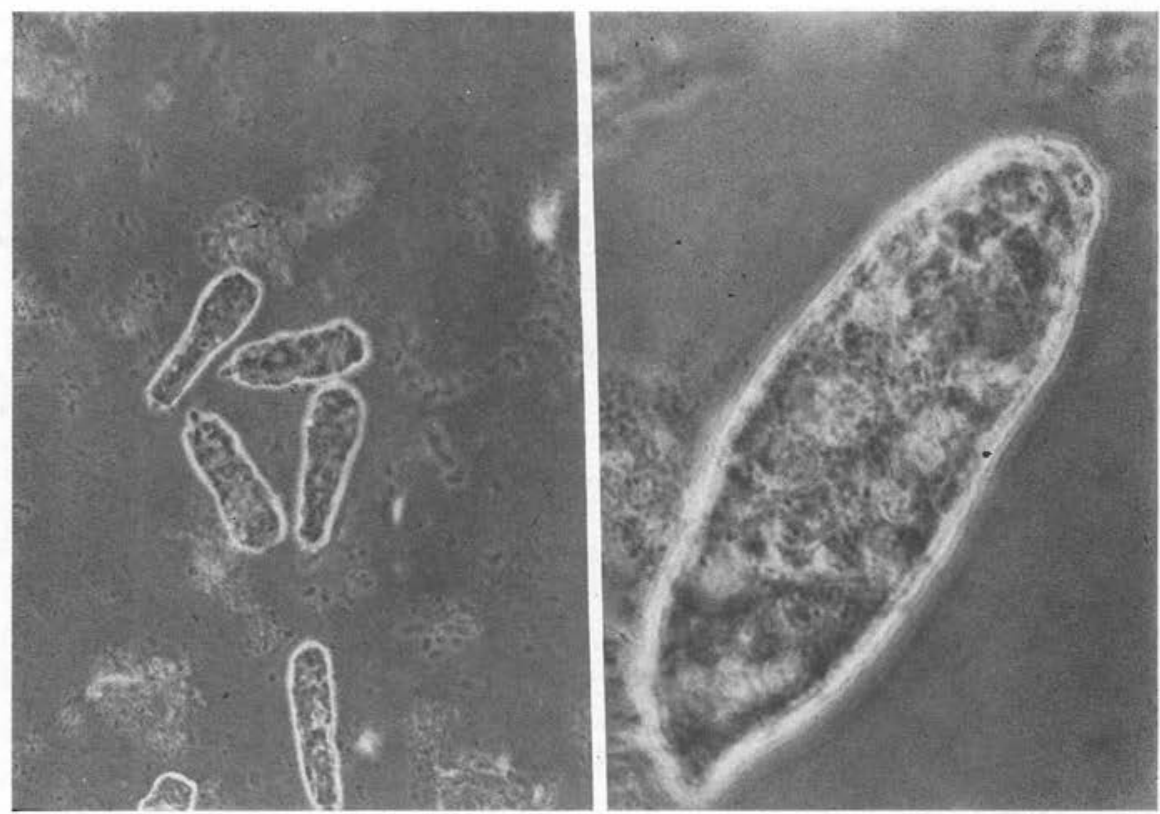

Fig. 1. Miracidiums après 2 heures de survie.

Fig. 2. Sporocystes après 16 jours de survie.

reste piriforme et fripé et aucune contraction n'est alors observée. Au bout de 48 heures, de tels miracidiums sont considérés comme morts.

Nos résultats sont supérieurs en durée (19 jours) et en degré d'évolution (30\% de sporocystes primaires) à ceux obtenus par Targett et Robinson. Par contre, ils sont plus proches de ceux rapportés par Basch et Diconza bien que l'expérimentation de ces auteurs ait une optique différente de la nôtre.

Par rapport aux 200 heures de Basch et Diconza et aux deux semaines maximum de Voge et Siegel, nous obtenons en règle générale 19 jours de survie et d'évolution parfaite; au-delà les sporocystes bien qu'encore vivants donnant des signes de souffrance et n'évoluant plus, nous considérons avoir atteint le terme de la survie. Nous 
comparons en effet l'aspect morphologique avec celui des sporocystes in situ dans le mollusque sans nous intéresser à l'ultrastructure. Notre but étant d'obtenir des stades évolutifs comparables à ceux décrits in vivo, afin de nous servir de ceux-ci pour des études ultérieures d'une réaction immunologique.

Nous n'avons pas obtenu une transformation en métasporocyste. Nous pensons que les milieux utilisés ne contiennent pas tous les éléments nécessaires à cette transformation. Des essais sont en cours pour mesurer l'influence d'extraits tissulaires (muscle ou hépato-pancréas) ajoutés au milieu.

De toutes façons, notre propos initial n'était pas d'obtenir l'évolution maximale mais seulement d'étudier le plus finement possible les réactions des miracidiums maintenus en milieu liquide. Ceci afin de confronter leur réaction en présence de cellules de mollusques éventuellement immunocompétentes.

Nous pouvons donc conclure que les miracidiums de Schistosoma mansoni fraîchement éclos sont capables de survivre et d'évoluer in vitro jusqu'au stade de sporocystes primaires parfaitement constitués avec un remaniement membranaire (perte des cils) comparable à celui qui survient in vivo (fig. 1 et 2).

\section{Bibliographie}

1. Basch P.F., Diconza J. J.: The miracidium sporocyst transition in Schistosoma mansoni: surfaces changes in vitro with ultrastructural correlation. J. Parasit., 1974, 60, 935-941.

2. Barlow C.H.: The life cycle human intestinal fluke Fasciolopsis buski. Am. J. Hyg., 1925, 4 (Monography).

3. Bénex J.: Les possibilités de la culture organo-typique en milieu liquide dans l'étude des problèmes parasitaires. I. La culture organo-typique d'explants de Planorbes. Ann. Parasitol., Hum. Comp., 1966, 41, 351-78.

4. Bénex J., Lamy L. : Immobilisation des miracidiums de $S$. mansoni par des extraits de Planorbes. Bull. Soc. Pathol. Exot., 1959, 52, 188.

5. Campbell W. C., Todd A. C.: In vitro metamorphosis of the miracidium of Fasciolopsis magna. Trans. Am. Micr. Soc., 1955, 74, 225.

6. Chernin E.: A method of securing bacteriogically sterile snails (Australorbis glabratus). Proc. Soc. Exp. Biol., 1957, 96, 204.

7. Ingersoll E. M. : In vitro survival of rediae of Cyclocoelum microstomum. Exp. Parasitol., 1956, $5,231$.

8. Maldonado J.F., Acosta Matienzo T. : Longevity and rates of infectivity of the free swimming miracidia of S. mansoni. J. Parasitol., 1947, 33, 331.

9. Schutte C. H. J.: Studies of the South Africa strain of S. mansoni. 2) the intramolluscan larval stages. 3) Notes on certain hosts parasit relationship. South Africa., J. Sci., 1974, 70, $327-46$ et $1975,71,8-19$.

10. Targett G. A. T., Robinson D.H. L. : Observations of the in vitro survival of miracidia of Schistosoma mansoni. Ann. Trop. Med. Parasitol., 1964, 58, 453-56.

11. Voge M., Seidel J.S.: Transformation in vitro of miracidia of Schistosoma mansoni and S. japo. nicum in young sporocysts. J. Parasitol., 1972, 58, 699-704. 